\title{
Current Perspectives on the Attainment of Lipid Modification Goals Relating to the Use of Statins and Ezetimibe for the Prevention of Cardiovascular Disease in the United Kingdom
}

Timothy Mark Reynolds'

Alison Pottle ${ }^{2}$

Sadat H Quoraishi $\mathbb{1 D}^{3}$

'University Hospitals of Derby \& Burton, Burton-on-Trent, UK; ${ }^{2}$ Royal Brompton \& Harefield NHS Foundation Trust, London, UK; ${ }^{3}$ Daiichi Sankyo UK Ltd, Uxbridge, UK
Correspondence: Timothy Mark Reynolds Pathology Department, Queen's Hospital, Belvedere Road, Burton-on-Trent. Staffordshire, DEI 3 ORB, UK

Tel +440I283 5II5II ext 4035

Email Timothy.Reynolds2@NHS.net

\begin{abstract}
Despite widespread evidence of the effectiveness of lipid modification for the reduction of cardiovascular disease (CVD) risk, lipid modification goals are commonly underachieved in the United Kingdom (UK). In order to understand current UK lipid management guidance and the corresponding attainment of recommended lipid lowering goals relating to treatment with statins and ezetimibe, a literature review was conducted using PubMed focusing on publications between January 2017 and February 2020 in order to capture the most up-to-date literature. Identified publications were reviewed against key clinical guidelines for lipid management in relation to CVD risk from the National Institute for Health and Care Excellence (NICE, CG181), the Scottish Intercollegiate Guidelines Network (SIGN, 149) and European Society of Cardiology (ESC)/European Atherosclerosis Society (EAS). Cholesterol lowering goals are central to current lipid lowering therapy guidance, although specific goals vary depending on the guideline and patients' individual risk profile. Current guidance by NICE and SIGN specifies that treatment should achieve a greater than $40 \%$ reduction in non-high-density lipoprotein cholesterol (non-HDLC) at 3 months of treatment, while the ESC/EAS place emphasis on the lowering of lowdensity lipoprotein (LDL-C) and total cholesterol. Yet, despite widespread availability of guidance and consistent messaging that lipid lowering goals should be ambitious, current evidence suggests a significant proportion of UK patients have sub-optimal reductions in cholesterol/non-HDL-C/LDL-C. The reasons for this are reported to be multifactorial, including a lack of compliance with guidelines, particularly regarding high-intensity statin prescribing, patient adherence, statin intolerance and statin reluctance as well as wider genetic factors. A number of possible strategies to improve current lipid management and attainment of lipid-lowering goals were identified, including improving the patient-healthcare professional partnership, conducting audits of local prescribing versus guidance, implementing plans for the refinement of current services and considering alternative options such as cost-effective single pill combinations for improving adherence.
\end{abstract}

Keywords: cholesterol, lipid management, guidelines, recommendations, cost effectiveness

\section{Introduction}

Cholesterol (including low-density lipoprotein cholesterol [LDL-C]), is a critical, modifiable risk factor in the prevention of cardiovascular disease (CVD). Based on well-established and long-term evidence, the lowering of LDL-C has emphatically been shown to reduce cardiovascular risk and all-cause mortality. ${ }^{1-5}$ However, 
Table I Research Search Terms

\begin{tabular}{|c|}
\hline $\mathbf{S}$ \\
\hline$((((($ statin OR statins OR ezetimibe)) AND (“cholesterol” OR “LDL-C” OR “LDL” OR “LDL-cholesterol” OR “lipid”)) AND (“treat to target” OR \\
\hline "treat-to-target" OR “target" OR "goal" OR "reduction")) AND ("achieve" OR "reach" OR "inadequate" OR "insufficient" OR "attain" OR \\
\hline 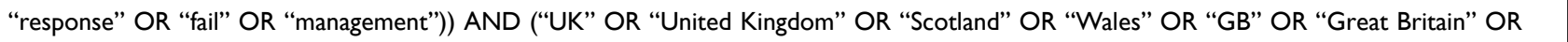 \\
\hline “England” OR “Northern Ireland” OR “NI”)) AND (“20I7/0I/0I”[Date - Create]: “2020/02/I8”[Date - Create]) \\
\hline
\end{tabular}

despite LDL-C being widely accepted as a causal cardiovascular risk factor and thus a critical component in CVD risk reduction, adequate lipid lowering is commonly underachieved in the United Kingdom (UK), creating a significant and unnecessary individual and societal burden in a population that accounts for almost one-third of deaths in England and Wales. ${ }^{6-9}$ This review article aims to summarize key UK lipid management guidance and outlines literature-derived recommendations which could be applied to improve UK attainment of cholesterol goals.

\section{Materials and Methods}

In order to understand current UK lipid management guidance in relation to treatment with statins and ezetimibe and the corresponding attainment of recommended lipid lowering goals, a literature review was conducted with PubMed using key search terms (as outlined in Table 1) and focusing on publications between January 2017 and
February 2020 in order to capture the most up-to-date literature. Figure 1 summarizes the literature search process and the number of publications excluded and reviewed. Only studies that evaluated lipid management in relation to statin and ezetimibe in the UK or at a global and/or European level (with either a large population size or UK sites) were included.

Abstracts with relevant titles were reviewed for keywords and those considered relevant to current UK practice were reviewed in full and additional publications were identified from the review of full-text article reference lists. Details of key studies that provided specific evidence of UK lipid modification in practice as well as recommendations for improved lipid modification were then extracted from each article. A large proportion of studies initially identified from the search were excluded due to their lack of relevance to the UK environment. Review of the reference lists of the applicable publications provided

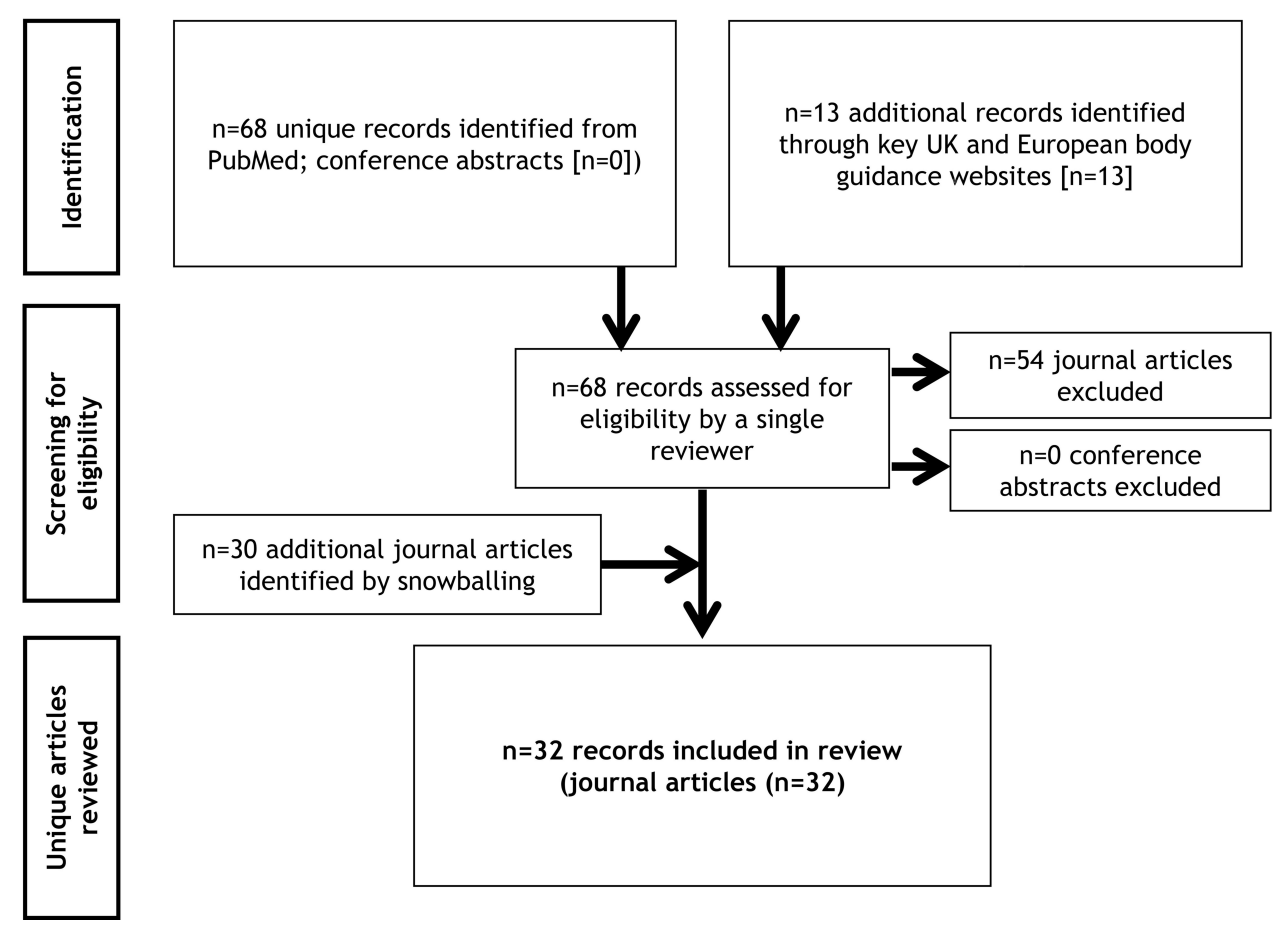

Figure I Flowchart of included publications for main literature review. 
further evidence of UK lipid modification in practice and insights into factors influencing compliance with guidelines.

In addition, in order to review current practice in light of UK best practice recommendations for lipid modification, key clinical guidelines issued by the National Institute for Health and Care Excellence (NICE), the Scottish Intercollegiate Guidelines Network (SIGN) and the European Society of Cardiology (ESC)/European Atherosclerosis Society (EAS) were identified and details of relevant recommendations extracted. While relevant for the UK, the Health and Social Care for Northern Ireland guidelines were not considered separately within this literature review due to the large degree of overlap with the NICE guidelines.

\section{Results \\ Best Practice Lipid Management in the UK}

The main clinical guidelines for lipid management in relation to cardiovascular risk in the UK are NICE CG181 (last updated in 2016) ${ }^{9}$ and SIGN 149 (2017). ${ }^{4}$

Figure 2 summarizes the key aspects of these guidelines. In addition to recommending lifestyle modifications (such as a cardioprotective diet, increased physical activity,

Lipid modification therapy

\section{Primary prevention}

A

Statin treatment 1,2

- Atorvastatin $20 \mathrm{mg}$ for $\geq 10 \%$ or greater 10 -year risk of developing CVD

- Estimate risk using the QRISK2 assessment tool

B Ezetimibe (hypercholesterolaemia)

- Monotherapy for primary (heterozygous-familial or non-familial) hypercholesterolaemia where initial statin therapy is contraindicated
Secondary prevention

Statin treatment 2

- Atorvastatin $80 \mathrm{mg}$ or at a lower dose for those with:

- Potential drug interactions OR at high risk of adverse events OR according to patient preference

Ezetimibe (hypercholesterolaemia)

- Monotherapy for primary (heterozygous-familial or non-familial) hypercholesterolaemia where initial statin therapy is contraindicated

Statin intolerance - If a person is not able to tolerate a high-intensity statin ${ }^{3}$ aim to treat with the maximum tolerated dose

C Follow up and monitoring

- Measure total cholesterol, HDL-C and non-HDL-C in all people who have been started on high-intensity statin treatment (both primary and secondary prevention, including atorvastatin $20 \mathrm{mg}$ for primary prevention) at 3 months of treatment and aim for a greater than $40 \%$ reduction in non-HDL-C For those who are stable on a low- or middle-intensity statin consider the likely benefits and potential risks of changing to a high-intensity statin

\section{$\downarrow$}

D Ezetimibe (primary hypercholesterolaemia) should be co-administered with initial statin therapy

Ezetimibe (primary hypercholesterolaemia) should be co-administered with initial statin therapy
- Serum total or LDL-C concentration ${ }^{4}$ is not appropriately controlled either after appropriate dose titration of initial statin therapy or because dose titration is limited by intolerance to the initial statin therapy and a change from initial statin therapy to an alternative statin is being considered

Evolocumab or alirocumab (primary hypercholesterolaemia or mixed dyslipidaemia) recommended as options only if:

LDL concentrations are persistently above the recommended LDL-C concentration thresholds despite maximal tolerated lipid-lowering therapy (e.g.

E in primary non-familial hypercholesterolaemia or mixed dyslipidaemia at high risk ${ }^{5}$ of CVD if persistently above $4.0 \mathrm{mmol} /$ litre OR at very high risk 6 if persistently above $3.5 \mathrm{mmol} /$ litre; in primary heterozygous-familial hypercholesterolaemia if LDL-C is persistently above $3.5 \mathrm{mmol} /$ litre) ie either the maximum dose has been reached, or further titration is limited by intolerance.

Figure 2 Summary of UK lipid modification guidance relating to the use of: (A) statins and (B) ezetimibe for the primary and secondary prevention of cardiovascular disease; (C) procedures for statin intolerance/follow up and monitoring; (D) ezetimibe co-administered with statin therapy and (E) additional options- evolocumab or alirocumab. [Adapted from NICE CGI8I, NICE pathway, SIGN I49]. ${ }^{4,9,43}$

Notes: I. For people with type I diabetes; offer statin treatment in those aged $\geq 40$ years OR with diabetes duration $>10$ years $O R$ with established nephropathy OR with CVD risk factors. 2. For people with chronic kidney disease: offer atorvastatin $20 \mathrm{mg}$ - increase the dose if a greater than $40 \%$ reduction in non-HDL cholesterol is not achieved (see follow-up and monitoring) and estimated glomerular filtration rate (eGFR) is $30 \mathrm{~mL} / \mathrm{min} / 1.73 \mathrm{~m}^{2}$ or more. Agree the use of higher doses with a renal specialist if eGFR is less than $30 \mathrm{~mL} / \mathrm{min} / 1.73 \mathrm{~m}^{2}$. 3. Statins are grouped into 3 different intensity categories according to the percentage reduction in LDL-C: low intensity if the reduction is from $20 \%$ to $30 \%$; medium intensity if the reduction is from $31 \%$ to $40 \%$; high intensity if the reduction is above $40 \%$ statin (aim to treat with the maximum tolerated dose). 4. Accurate estimation of LDL-C requires a full lipid profile to be carried out on a fasting venous blood sample as HDL-C and triglyceride (TG) levels vary between fasting and non-fasting states. However, LDL-C can be calculated indirectly by measuring total cholesterol (TC), HDL-C and TG from a fasting venous blood sample and applying the Friedewald equation: $\mathrm{LDL}=\mathrm{TC}-\mathrm{HDL}-(\mathrm{TG} / 2.2)$ (all in $\mathrm{mmol} / \mathrm{l})$ where $\mathrm{TG} / 2.2$ approximates to very low-density lipoprotein cholesterol levels. This method is not suitable for individuals with TG levels $>4.5 \mathrm{mmol} / \mathrm{l}$. Non-HDL-C is calculated as TC-HDL. 5. High risk of CVD is defined as a history of any of the following: acute coronary syndrome, coronary or other arterial revascularisation procedures, coronary heart disease, ischaemic stroke, peripheral arterial disease. 6 . Very high risk of CVD is defined as recurrent cardiovascular events or cardiovascular events in more than one vascular bed (ie polyvascular disease).

Abbreviations: CVD, cardiovascular disease; HDL-C, high-density lipoprotein cholesterol; LDL-C, low-density lipoprotein cholesterol. 
smoking cessation and reduction in alcohol consumption), current UK guidelines advise that lipid lowering therapy should be considered for both the primary and secondary prevention of CVD based on an assessment of 10-year risk of developing CVD. A range of lipid modifying therapies have been approved for use in the UK, with statins being the mainstay of initial recommended treatment strategies (eg it is currently recommended that high-intensity statin [80mg atorvastatin] should be prescribed for secondary prevention of CVD; Figure 2A). Extensive randomized controlled trials and meta-analyses have shown that statins improve lipid profiles in men and women across all age groups over prolonged periods of time. ${ }^{2,3,10,11}$ Statins are typically classified as low, medium and high intensity, according to the percentage reduction in LDL-C that can be achieved, with current recommendations advising the use of high-intensity statins where possible to expedite target attainment. ${ }^{4,9}$ Alternatively, ezetimibe monotherapy is recommended for both primary and secondary CVD prevention where initial statin therapy is contraindicated for adults with primary (heterozygous-familial or nonfamilial) hypercholesterolemia or in combination with initial statin therapy when cholesterol levels are not appropriately controlled (Figure 2B and D). ${ }^{4,9}$

Current guidelines place weight on lipid profiles for the assessment of cardiovascular risk prior to starting lipid lowering therapy. The most recent iteration of the NICE and SIGN clinical guidelines emphasizes the importance of individualized risk assessment (using QRISK score where appropriate) and management by recommending that a full lipid profile including total cholesterol, highdensity lipoprotein cholesterol (HDL-C), non-HDL-C and triglyceride concentrations is evaluated prior to initiating lipid lowering therapy in order to best determine cardiovascular risk and to identify circumstances where additional support may be needed. ${ }^{4,9}$ For example, NICE states that specialist assessment will be required for people with a total cholesterol concentration of more than $9.0 \mathrm{mmol} /$ liter or a non-HDL-C concentration of more than $7.5 \mathrm{mmol} /$ liter, irrespective of family history of premature coronary heart disease. The current NICE guidance also specifies that "urgent specialist review" will be required for any individual with a triglyceride concentration of more than $20 \mathrm{mmol} /$ liter that is not a result of excess alcohol or poor glycemic control. ${ }^{9}$ It is also recommended that the use of higher statin doses should be agreed with a renal specialist for patients with chronic kidney disease (CKD). ${ }^{9}$ The guidance does not, however, provide further detail about the definition of a specialist or the specific types of support that should be accessed in particular situations, and associated processes for accessing this. Once patients are initiated on high-intensity statin treatment NICE guidance recommends that they should be followed up at three months in order to monitor the effectiveness of lipid lowering therapy and make further adaptations to treatment where required. These adaptations may include increasing dose for high-risk patients or changing the timing of doses, as well as encouraging the implementation of other strategies for improving adherence and wider diet and lifestyle factors. ${ }^{9}$

Guidance is also beginning to consider the importance of patient preferences for treatment decisions. ${ }^{4,9} \mathrm{NICE}$ guidance emphasizes that the decision to initiate statin therapy should be informed by discussion between the clinician and the patient about treatment and the associated risks and benefits, which also takes additional factors into consideration such as potential benefits from lifestyle modifications, informed patient preference, comorbidities, polypharmacy, general frailty and life expectancy. ${ }^{9}$ This discussion should be an ongoing process, and medication reviews are recommended annually for people taking statins, alongside optional non-fasting blood tests for monitoring the effectiveness of lipid lowering therapy to inform the discussion. ${ }^{4,9}$

While the full lipid profile is used as the basis for decisions to initiate statin therapy, UK guidance specifies that attainment of specific cholesterol goals should be used to determine whether lipid lowering therapy should be altered or changed in order to reach treatment goals (Figure 2C). While traditional approaches for monitoring the effectiveness of lipid lowering therapy have targeted LDL-C and total cholesterol levels, UK lipid modification guidance has since moved away from primarily focusing on LDL-C in favor of non-HDL-C, in part due to the fasting requirements for accurate measurement of LDLC. ${ }^{9}$ Specifically, NICE and SIGN guidance recommend that lipid lowering therapy should achieve a greater than $40 \%$ reduction in non-HDL-C at three months of treatment (Figure 2C). ${ }^{4,9}$ In contrast, alternative approaches, such as direct and calculated measures of LCL-C to assess risk, remain commonplace across global centers and laboratories. The European ESC/EAS guidelines for the management of dyslipidemias continue to place emphasis on lowering LDL-C and total cholesterol as the primary target of therapy. ${ }^{9,12,13}$ In August 2019, the ESC/EAS guidelines were updated to recommend more aggressive reduction of 
LDL-C across different cardiovascular risk categories: for example, for certain patients deemed to be at very high risk, an LDL-C reduction of $\geq 50 \%$ from baseline and an LDL-C goal of $<1.4 \mathrm{mmol} /$ liter $(<55 \mathrm{mg} /$ deciliter) are recommended. ${ }^{13}$ Therefore, while the different guidelines are aligned on the requirement to reduce lipid levels as much as possible, the specific goals being aimed for may vary depending on the guideline being followed within routine practice.

Despite the importance of non-HDL-C and the wider lipid profile for decision-making relating to the effectiveness of initial lipid lowering therapy, LDL-C measurement in the UK remains relevant in routine clinical practice since guidance for later lines of therapy (post-statin) is predicated on evaluation of LDL-C levels. ${ }^{9}$ For example, NICE states that ezetimibe should be considered in combination with statin therapy for the treatment of primary hypercholesterolemia in situations where total cholesterol or LDL-C is not appropriately controlled after initial statin therapy (Figure 2B and D). ${ }^{9,14}$ Likewise, alternative therapies such as the proprotein convertase subtilisin/kexin type 9 (PCSK9) inhibitors evolocumab or alirocumab may be considered for certain patients with hypercholesterolemia or mixed dyslipidemia who have high or very high risk of CVD, but only if LDL-C levels are persistently above recommended thresholds (Figure 2E). ${ }^{9}$ The most recent ESC/EAS guidelines also state that should patients fail to reach their LDL-C goal (eg $\geq 50 \%$ reduction from baseline and LDL-C levels of $<1.4 \mathrm{mmol} /$ liter [very high risk] or $<1.8 \mathrm{mmol} /$ liter [high risk]) within four to six weeks despite lifestyle modifications and treatment with maximally tolerated high-intensity statins, then add-on therapy with ezetimibe and subsequently a PCSK9 inhibitor should be considered. ${ }^{13}$ A referral to a specialist with expertise in familial hypercholesterolaemia should also be offered to adults with familial hypercholesterolaemia if treatment with the maximum tolerated dose of a high-intensity statin and ezetimibe does not achieve a recommended reduction in LDL-C concentration of greater than $50 \%$ from baseline. $^{15}$

While much of existing UK guidance is focused on the importance of lowering lipid levels using lipid lowering therapy along with other non-interventional strategies (such as diet, activity, lifestyle changes) to reduce cardiovascular risk, ongoing management should also be informed by supplementary means of assessing treatment effectiveness, where available. For example, the latest ESC/EAS 2019 guidance acknowledges that additional approaches, such as utilizing non-invasive cardiovascular imaging to detect and evaluate atherosclerotic vascular damage, could be effective for identifying patients who most need treatment and to guide discussions around longterm statin therapy. ${ }^{13}$

Statin intolerance, whereby patients are unable to tolerate (eg due to adverse events) the statin treatment dose needed to control LDL-C levels, is another important consideration for lipid modification therapy. The most recent iterations of both the NICE and SIGN guidance specify that patients who are unable to tolerate high-intensity statins should be treated with the maximum tolerated dose, on the grounds that "any statin at any dose reduces cardiovascular risk". 4,9 Strategies for addressing intolerance include stopping the statin and restarting when the symptoms have resolved, reducing the dose within the same intensity group or switching to a lower intensity statin. ${ }^{9}$ Current UK guidance also recommends that specialist input regarding alternate treatment options (currently limited to ezetimibe or PCSK9 inhibitors) is gained for patients at high risk of developing CVD (such as patients with chronic kidney disease, type 1 or type 2 diabetes or genetic dyslipidemia) if they have been found to be intolerant to three different statins. ${ }^{4,9}$ Specialist advice should also be sought for patients with elevated triglyceride concentrations, if triglyceride and non-HDL concentrations remain high despite treatment at the recommended dose. ${ }^{4,9}$ However, there is currently no widely used, standardized definition of statin intolerance and how to monitor it, to guide decision-making in UK practice.

\section{Sub-Optimal Achievement of Guideline- Derived Lipid Modification Goals}

Despite widespread availability and comprehensive evidence supporting the effectiveness of statins and combination therapy with ezetimibe, a number of large UK studies evaluating the effectiveness of lipid lowering therapy in patients with or at risk of developing CVD have highlighted that a significant proportion of patients are not being adequately managed according to best practice guidelines. $^{7,8,16-19}$ For example, a study evaluating a cohort of 165,411 people using UK Clinical Practice Research Datalink (CPRD) ${ }^{7}$ data reported that over half of those prescribed statins in primary care failed to reach a $>40 \%$ reduction in baseline LDL-C within 24 months of initiating therapy, leaving them at an increased risk for 
future CVD. ${ }^{7}$ It was suggested that variations in individual patient genotypes, statin intolerance, patient adherence and the potency of statin prescribing might partially explain the high proportion of patients achieving sub-optimal LDL-C reductions. ${ }^{7}$ A number of large global and European studies have also reported sub-optimal LDL-C management, ${ }^{6,20}$ with a relatively high proportion of patients receiving reduced intensity dosing or interruptions to lipid lowering therapy. ${ }^{6}$ Indeed, a lack of prescribing of high-intensity statin therapy in high-risk patients was a reoccurring theme across both UK and wider studies. ${ }^{8,16,17}$ This finding may in part be related to a general lack of awareness of guidelines and/or an inadequate level of formal CVD risk assessment to inform treatment eligibility. ${ }^{6,18}$ Limited prescribing of combination therapy may also contribute to sub-optimal lipid lowering. For example, both the UK and European guidelines recommend that ezetimibe is used alongside statin therapy in patients at high risk of CVD if lipid lowering goals are not achieved with the maximum tolerated dose of statin, or in cases where statin cannot be prescribed. ${ }^{6,13,14}$ Yet, despite the known benefits of adding ezetimibe, particularly for patients remaining at high atherothrombotic risk (eg postmyocardial infarction), ${ }^{13}$ rates of ezetimibe utilization as combination therapy in real-world clinical practice appear to be low. For example, one study reported that only $2.7 \%$ of patients had received a high-intensity statin with ezetimibe. $^{6}$

In addition to the above evidence, the identified literature further highlighted other factors likely to contribute to sub-optimal lipid lowering. These included late intervention in the disease trajectory, ${ }^{21}$ a lack of follow-up and treatment adaptation according to risk factors or fulfilment of therapeutic goals, and statin intolerance. ${ }^{8}$ While generally well-tolerated by patients, it is known that statins can lead to specific adverse effects on muscles, and other adverse events such as impaired glucose homeostasis and hemorrhagic stroke in some patients. However, it has been suggested that estimates of the frequency of statin-related adverse events, such as those relating to muscle symptoms, may be overestimated due to an apparent "nocebo" effect, whereby patients' perceptions are influenced by negative expectations of treatment. ${ }^{13}$ Therefore, misconceptions around statin intolerance and statin-related adverse events may lead to a reluctance to take statins that may result in early discontinuation and/or non-adherence to treatment and inadequate lipid lowering. ${ }^{19,22}$

\section{Possible Strategies to Improve Current Lipid Management}

It is clear that there remains significant scope to improve lipid management of patients in the UK and consequently improve outcomes in what is the UK's leading cause of death. ${ }^{9}$ Some possible strategies and potential areas for focus highlighted in the literature are summarized in Table 2. These approaches include measures for improving the patient-healthcare professional partnership, conducting audits of local prescribing versus guidance and implementing plans for the refinement of current services including referral, follow-up and monitoring procedures for different types of therapy, and considering alternative options such as cost-effective single pill for improving treatment adherence.

\section{Discussion}

\section{Future Considerations}

This review identified some areas for improvement regarding the attainment of adequate lipid management in practice in light of the UK clinical guidance. The UK studies identified demonstrate that there is first and foremost scope for improved compliance with guidance, particularly in terms of high-intensity statin prescribing, utilization of combination therapies and regular patient monitoring. However, while current UK and European guidance emphasizes the importance of setting ambitious goals for lipid reduction as early as possible, the specific goals for lipid reduction vary depending on the guideline in question and the individual risk of patients. The large volume of guidelines available for consideration (particularly for general practitioners) as well as some inconsistencies regarding the specific recommendations (such as the exact lipid lowering goals to be aimed for and the emphasis placed on non-HDL-C versus LDL-C) may have led to variability in clinical practice regarding setting specific lipid lowering goals, which may in part have contributed to discrepancies in research outcomes. This possibility is likely to be particularly pertinent for observational research studies based on retrospective data collection from medical records, where the guideline being followed and/or the individual patient goals may not have been adequately recorded within medical records. Furthermore, while current guidance specifies situations where specialist support should be sought, there is presently no specific definition of a "specialist" within the guidelines. ${ }^{9}$ The subsequent interpretation of this guidance and associated referral processes 


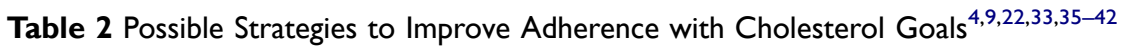

\begin{tabular}{|c|c|}
\hline $\begin{array}{l}\text { Possible Cause(es) for Failure to Meet } \\
\text { Cholesterol Goals }\end{array}$ & Possible Strategies/Solution(s) \\
\hline Underdosing of statins & $\begin{array}{l}\text { - Audit/benchmark local prescribing versus guidance to identify gaps and fill need via medical } \\
\text { education } \\
\text { - Incorporate risk calculators/prediction more comprehensively into everyday practice } \\
\text { - Gain specialist advice and/or refer patients where appropriate (will require clear guidance as to } \\
\text { definition of "specialist support" and how to access this) }\end{array}$ \\
\hline Treatment initiated too late & $\begin{array}{l}\text { - Incorporate a systematic case finding strategy to identify patients }{ }^{43} \\
\text { - Utilize additional measures such as non-invasive cardiovascular imaging to identify patients who } \\
\text { may require treatment at an earlier stage (including those who are otherwise deemed to be at } \\
\text { low or moderate risk) }\end{array}$ \\
\hline Adherence & $\begin{array}{l}\text { Work to improve adherence as follows: } \\
\text { - Ensure screening for poor medication adherence is integral to each patient visit as adherence } \\
\text { may vary with time }{ }^{21} \\
\text { - Provide patient counselling and education at the onset of treatment, including discussion about } \\
\text { the overall safety, risks and benefits of treatment, }{ }^{9} \text { to ensure the patient is supported and } \\
\text { understands the risk of CVD events }{ }^{37,44} \\
\text { - Cost-effective single pill combinations }{ }^{21}\end{array}$ \\
\hline Statin intolerance AND/OR reluctance & $\begin{array}{l}\text { - Make the distinction between statin intolerance and statin reluctance. Rosenson et al and Banach } \\
\text { and Mikhailidis outline a four-step diagnosis of statin intolerance as follows: }{ }^{22,38} \\
\text { Confirm when statin therapy was initiated or if there has been a recent dose increase } \\
\text { Obtain a family history and identify any conditions that could contribute to statin intolerance } \\
\text { - Exclude nocebo effect and confirm if any muscle symptoms could be attributed to statin } \\
\text { therapy } \\
\text { - Discuss symptom tolerability whilst underlining the benefits and risks associated with statin } \\
\text { treatment vs discontinuation } \\
\text { - For statin intolerance: } \\
\text { Consider patient suitability for alternative treatment (eg ezetimibe and/or PSCK9 inhibitors) } \\
\text { and other new agents }{ }^{9} \text { and identify and address any barriers to access (may include imple- } \\
\text { mentation of new dedicated services to improve access }{ }^{33} \text { ) } \\
\text { To address potential statin reluctance: } \\
\text { Before commencing therapy, have an open discussion with patients about existing effective- } \\
\text { ness and safety data for statin therapy and explore any concerns patient may have } \\
\text { - Aim to involve patients in the awareness of lipid goals and in decisions regarding change of } \\
\text { therapy } \\
\text { Ongoing communication: discuss risks, take note of patient preferences and reassess at later } \\
\text { date }{ }^{4} \\
\text { Utilize available toolkits to aid discussions with patients such as the patient decision aid toolkit } \\
\text { developed by NICE }{ }^{44} \\
\text { Utilize additional measures such as non-invasive imaging to detect and monitor damage, and } \\
\text { guide ongoing discussions about risk and subsequent statin therapy }{ }^{13}\end{array}$ \\
\hline $\begin{array}{l}\text { Lack of systematic follow up AND/OR } \\
\text { patient personalization }\end{array}$ & $\begin{array}{l}\text { - Audit current follow up/monitoring of lipid lowering therapy in local practice and identify gaps to } \\
\text { improve standard practice AND/OR } \\
\text { - Investigate different interventions to target issues with CVD risk factors and/or adherence eg } \\
\text { nurse-led, multidisciplinary approach community interventions or secondary care multi-disci- } \\
\text { plinary medicines optimization clinics }{ }^{33,39} \\
\text { - Personalize treatment according to the individual relative risk - generate a detailed CV event } \\
\text { history to support }\end{array}$ \\
\hline
\end{tabular}

(Continued) 
Table 2 (Continued).

\begin{tabular}{|l|l|}
\hline $\begin{array}{l}\text { Possible Cause(es) for Failure to Meet } \\
\text { Cholesterol Goals }\end{array}$ & \multicolumn{1}{c|}{ Possible Strategies/Solution(s) } \\
\hline Inadequate response to statin AND/OR \\
combination statin/ezetimibe & $\bullet \begin{array}{l}\text { Measure statin response more proactively and, where appropriate in poor responders, consider } \\
\text { more aggressive therapy either with lipid lowering therapy, diet or improved adherence }\end{array}$ \\
& $\begin{array}{l}\text { Consider patient eligibility for combined therapy (eg adding ezetimibe to statin therapy), or other } \\
\text { non-statin therapies (eg PCSK9 inhibitors or other newly licensed agents) for those on maximum } \\
\text { tolerated statin dose or when absolute risk requires it (eg LDL-C }>4 \text { mmol/liter) }\end{array}$ \\
\hline 4,42
\end{tabular}

may therefore vary across different centers, which may in turn lead to variation in practice and outcomes. Further research describing the utilization of specific guidelines for defining lipid lowering goals within current practice may therefore help to stimulate future discussion about the most appropriate approaches for monitoring and modification of lipid lowering therapy to minimize future CVD risk.

In addition to variability across different guidelines in terms of lipid lowering goals, an awareness of differences in the methods used for the assessment of lipid levels is also of importance. For example, while LDL-C is often the main target of treatment, there may be variation in how this is assessed. For example, some laboratories may utilize a specific algorithm to calculate LDL-C levels instead of direct measures, in part due to the associated complexity and cost of direct assessment. ${ }^{23} \mathrm{~A}$ number of equations for calculation of LDL-C are used in practice, most of these never having been validated for patients on statins or other lipid lowering drugs, ${ }^{23}$ which may in turn lead to variation in LDL-C levels across practices.

Given the findings of this current review, it is important to recognize that for certain patients there will be a need for additional lipid lowering therapy in cases where the response to the maximally tolerated statin dose has been inadequate. ${ }^{24}$ The rate of discontinuation of statin therapy once initiated is high, meaning that a large proportion of patients likely remain at increased risk of cardiac events after initiating lipid lowering therapy ${ }^{25}$ and require alternative treatment options, such as PCSK9 inhibitors or other non-statin agents that target LDL cholesterol via novel mechanisms. The review also highlighted the particular complexities associated with the management of patients who cannot tolerate maximal doses of statin, meaning many of these patients remain at risk of further $\mathrm{CV}$ events. Further research about current practices for the detection and management of statin intolerance in realworld practice may help to move towards a more standardized definition and the refinement of associated processes for LDL management. Furthermore, there appear to be further questions around accessibility and eligibility for alternative or additional treatments for some patients who are deemed to have achieved an inadequate response to maximally tolerated statin doses. NICE eligibility criteria for evolocumab or alirocumab for patients with primary non-familial hypercholesterolemia or mixed dyslipidemia states that patients must have LDL-C levels that persist above $3.5 \mathrm{mmol} /$ liter for very high-risk patients (ie patients with recurrent cardiovascular events) or above $4.0 \mathrm{mmol} /$ liter for high-risk patients (ie patients with a history of acute coronary syndrome, coronary or other arterial revascularization procedures, coronary heart disease, ischemic stroke or peripheral arterial disease), despite maximal tolerated lipid lowering therapy. ${ }^{26-28}$ Yet in reality, many patients with sub-optimal responses to statins according to guidelines may not have reached these thresholds, even if guidance regarding the maximization of statin dose has been fully adhered to. Some evidence suggests that there is a sizeable subset of patients who are currently "in limbo" in that they have been unable to gain adequate LDL-C control using statin therapy but have not received non-statin therapies. ${ }^{26}$ Some of these patients might benefit from the addition of nonstatin treatments such as ezetimibe to their existing statin therapy (if tolerated) or the direct initiation of PCSK9 inhibitors ${ }^{26}$ or indeed other newly licensed therapies such as bempedoic acid $^{29}$ or inclisiran ${ }^{30}$ that are emerging in the treatment landscape. Furthermore, there may be other barriers to accessing treatment, such as price or administrative burden, that should be identified and addressed. ${ }^{26,31}$

Failure to attain cholesterol/non-HDL-C/LDL-C reduction goals may also be attributed to a variety of other factors, including poor adherence and statin intolerance, which could require alternative approaches in order to personalize patient care and minimize CVD risk. Healthcare professionals may, therefore, benefit from improved 
and validated clinical decision tools that can be used to predict response and support clinicians to identify patients most likely to benefit from combinations with statin therapy or alternative non-statin drugs. ${ }^{7}$ For example, there may be a need for further research and refined protocols for identifying the drivers of statin resistance or intolerance to inform management, and more detailed guidance on options for further treatment.

Additional strategies for improving access to alternative therapies might also include the introduction of specialist services and clinics that can use PCSK9 inhibitors, and/or act as early adopters of new agents, in order to increase knowledge and awareness of new treatments among prescribers within both primary and secondary care. $^{32,33}$ The utilization of additional measures such as non-invasive cardiovascular imaging may also help facilitate ongoing discussions about optimal treatment strategies between clinicians and their patients in the context of addressing issues relating to statin reluctance and adherence. ${ }^{13}$ It will also be important to directly address key drivers of the "nocebo effect", which may also influence levels of adherence to therapy and subsequent decisions to discontinue treatments. ${ }^{34}$

\section{Conclusion}

Despite widespread availability of lipid lowering therapy and associated UK guidelines, a significant proportion of UK patients with CVD have sub-optimal reductions in cholesterol/non-HDL-C/LDL-C. Although specific lipid lowering goals vary depending on the individual risk of patients and the clinical guideline(s) adopted in routine clinical practice, failure to achieve lipid lowering goals can, at least in part, be attributed to a lack of compliance with guideline recommendations. This review highlights various strategies for improving lipid management in order to reduce CVD risk. Additional options are required to manage and treat patients at high risk of CVD events, where lipid lowering goals are not attained with statins and ezetimibe alone.

\section{Abbreviations}

CVD, cardiovascular disease; ESC/EAS, European Society of Cardiology/European Atherosclerosis Society; HDL-C, high-density lipoprotein cholesterol; LDL-C, lowdensity lipoprotein cholesterol; NICE, National Institute of Health and Care Excellence; PCSK9, proprotein convertase subtilisin/kexin type 9; SIGN, Scottish Intercollegiate Guidelines Network; UK, United Kingdom.

\section{Acknowledgments}

Medical writing support for this literature review and manuscript was provided by OPEN VIE (Nicole Anderson and Fiona Glen).

\section{Disclosure}

The development of this manuscript was initiated and funded by Daiichi Sankyo UK, who were involved in the design of the literature review and the review and approval of the manuscript.

- TR has received funding to attend conferences from Sanofi-Genzyme and for research projects (investigatorled and multicentre trials) from Alexion Pharma UK Ltd, AmGen Ltd, Boehringer Ingelheim $\mathrm{GmbH}$, Dalcor Pharmaceuticals, Sanofi-Genzyme, Shire Pharmaceuticals Ltd, Takeda Ltd. He has no direct stock holdings in any pharmaceutical company or any patents.

- AP has received funding to attend conferences from Sanofi.

- SHQ is an employee of Daiichi Sankyo UK Ltd. The parent company Daiichi Sankyo Europe GmbH are marketing authorisation holders of bempedoic acid and the bempedoic acid/ezetimibe combination in the UK, European Economic Area and Switzerland.

\section{References}

1. Ference BA, Ginsberg HN, Graham I, et al. Low-density lipoproteins cause atherosclerotic cardiovascular disease. 1. Evidence from genetic, epidemiologic, and clinical studies. A consensus statement from the European Atherosclerosis Society Consensus Panel. Eur Heart J. 2017;38(32):2459-2472. doi:10.1093/eurheartj/ehx144

2. Alkhalil M. Effects of intensive lipid-lowering therapy on mortality after coronary bypass surgery: a meta-analysis of 7 randomised trials. Atherosclerosis. 2020;293:75-78. doi:10.1016/j.atheroscleros is.2019.12.006

3. Armitage J, Baigent C, Barnes E, Cholesterol Treatment Trialists' Collaboration. Efficacy and safety of statin therapy in older people: a meta-analysis of individual participant data from 28 randomised controlled trials. Lancet. 2019;393(10170):407-415. doi:10.1016/S01406736(18)31942-1

4. Scottish Intercollegiate Guidelines Network (SIGN). SIGN 149. Risk estimation and the prevention of cardiovascular disease. 2017. Available from: https://www.sign.ac.uk/assets/sign149.pdf. Accessed April 29, 2021.

5. Giugliano RP, Pedersen TR, Park J-G, et al. Clinical efficacy and safety of achieving very low LDL-cholesterol concentrations with the PCSK9 inhibitor evolocumab: a prespecified secondary analysis of the FOURIER trial. Lancet. 2017;390(10106):1962-1971. doi:10.1016/S0140-6736(17)32290-0

6. De Backer G, Jankowski P, Kotseva K, et al. Management of dyslipidaemia in patients with coronary heart disease: results from the ESCEORP EUROASPIRE V survey in 27 countries. Atherosclerosis. 2019;285:135-146. doi:10.1016/j.atherosclerosis.2019.03.014 
7. Akyea RK, Kai J, Qureshi N, Iyen B, Weng SF. Sub-optimal cholesterol response to initiation of statins and future risk of cardiovascular disease. Heart. 2019;105(13):975-981. doi:10.1136/heartjnl-2018314253

8. Danese MD, Gleeson M, Kutikova L, et al. Management of lipidlowering therapy in patients with cardiovascular events in the UK: a retrospective cohort study. BMJ Open. 2017;7(5):e013851. doi:10.1136/bmjopen-2016-013851

9. National Institute for Health and Care Excellence (NICE). Cardiovascular disease: risk assessment and reduction, including lipid modification. CG181. Available from: www.nice.org.uk/gui dance/cg181. Accessed April 29, 2021.

10. Vallejo-Vaz AJ, Robertson M, Catapano AL, et al. Low-density lipoprotein cholesterol lowering for the primary prevention of cardiovascular disease among men with primary elevations of low-density lipoprotein cholesterol levels of $190 \mathrm{mg} / \mathrm{dL}$ or above: analyses from the WOSCOPS (West of Scotland Coronary Prevention Study) 5-year randomized trial and 20-year observational follow-up. Circulation. 2017;136(20):18781891. doi:10.1161/CIRCULATIONAHA.117.027966

11. Karlson BW, Palmer MK, Nicholls SJ, Barter PJ, Lundman P. Effects of age, gender and statin dose on lipid levels: results from the VOYAGER meta-analysis database. Atherosclerosis. 2017;265:5459. doi:10.1016/j.atherosclerosis.2017.08.014

12. Catapano AL, Graham I, De Backer G, et al. 2016 ESC/EAS guidelines for the management of dyslipidaemias. Eur Heart J. 2017;70 (2):115. doi:10.1016/j.rec.2017.01.002

13. Mach F, Baigent C, Catapano AL, et al. 2019 ESC/EAS guidelines for the management of dyslipidaemias: lipid modification to reduce cardiovascular riskThe task force for the management of dyslipidaemias of the European Society of Cardiology (ESC) and European Atherosclerosis Society (EAS). Eur Heart J. 2020;41(1):111-188. doi:10.1093/eurheartj/ehz455

14. Overview. Ezetimibe for treating primary heterozygous-familial and non-familial hypercholesterolaemia. Guidance. NICE. Available from: https://www.nice.org.uk/guidance/ta385. Accessed April 29, 2021.

15. Familial hypercholesterolaemia: identification and management: NICE guidance [CG71]. Available from: https://www.nice.org.uk/gui dance/cg71/chapter/Recommendations. Accessed December 4, 2020.

16. Saratzis A, Jaspers NEM, Gwilym B, et al. Observational study of the medical management of patients with peripheral artery disease. $\mathrm{Br} \mathrm{J}$ Surg. 2019;106(9):1168-1177. doi:10.1002/bjs.11214

17. Nordstrom B, Collins J, Donaldson R, Tockhorn A, Zhu Y, Zhao Z. Treatment patterns and lipid levels among patients with high-risk atherosclerotic CVD in the UK. Br J Cardiol. 2015;22:147-154. doi:10.5837/bjc.2015.034

18. Finnikin S, Ryan R, Marshall T. Statin initiations and QRISK2 scoring in UK general practice: a THIN database study. $\mathrm{Br} J \mathrm{Gen}$ Pract. 2017;67(665):e881-e887. doi:10.3399/bjgp17X693485

19. Matthews A, Herrett E, Gasparrini A, et al. Impact of statin related media coverage on use of statins: interrupted time series analysis with UK primary care data. BMJ. 2016;353:i3283. doi:10.1136/bmj.i3283

20. Ferrieres J, De Ferrari GM, Hermans MP, et al. Predictors of LDLcholesterol target value attainment differ in acute and chronic coronary heart disease patients: results from DYSIS II Europe. Eur $J$ Prev Cardiol. 2018;25(18):1966-1976. doi:10.1177/ 2047487318806359

21. Williams B, Masi S, Wolf J, Schmieder RE. Facing the challenge of lowering blood pressure and cholesterol in the same patient: report of a symposium at the European Society of hypertension. Cardiol Ther. 2020;9(1):19-34. doi:10.1007/s40119-019-00159-1

22. Banach M, Mikhailidis DP. Statin intolerance: some practical hints. Cardiol Clin. 2018;36(2):225-231. doi:10.1016/j.ccl.2017.12.004

23. Preiss D, Neely D. Biochemistry laboratories should routinely report non-HDL-cholesterol. Ann Clin Biochem. 2015;52(6):629-631. doi: $10.1177 / 0004563215594818$
24. Soran H, Dent R, Durrington P. Evidence-based goals in LDL-C reduction. Clin Res Cardiol. 2017;106(4):237-248. doi:10.1007/ s00392-016-1069-7

25. Toth PP, Banach M. Statins: then and now. Methodist DeBakey Cardiovasc J. 2019;15(1):23-31. doi:10.14797/mdcj-15-1-23

26. Elamin AFM, Grafton-Clarke C, Wen Chen K, et al. Potential use of PCSK9 inhibitors as a secondary preventative measure for cardiovascular disease following acute coronary syndrome: a UK real-world study. Postgrad Med J. 2019;95(1120):61-66. doi:10.1136/postgradmedj-2018-136171

27. Overview. Evolocumab for treating primary hypercholesterolaemia and mixed dyslipidaemia. Guidance. NICE. Available from: https:// www.nice.org.uk/guidance/ta394. Accessed April 29, 2021.

28. Overview. Alirocumab for treating primary hypercholesterolaemia and mixed dyslipidaemia. Guidance. NICE. Available from: https:// www.nice.org.uk/guidance/ta393. Accessed April 29, 2021.

29. National Institute for Health and Care Excellence. British National Formulary: indications, dose, contra-indications, side-effects, interactions, cautions, warnings and other safety information for BEMPEDOIC ACID. Available from: https://bnf.nice.org.uk/drug/ bempedoic-acid.html. Accessed January 21, 2021.

30. European Medicines Agency. Leqvio. European Medicines Agency. October 13, 2020. Available from: https://www.ema.europa.eu/en/ medicines/human/EPAR/leqvio. Accessed January 21, 2021.

31. Cohen JD, Cziraky MJ, Jacobson TA, Maki KC, Karalis DG. Barriers to PCSK9 inhibitor prescriptions for patients with high cardiovascular risk: results of a healthcare provider survey conducted by the National Lipid Association. J Clin Lipidol. 2017;11(4):891-900. doi:10.1016/j.jacl.2017.04.120

32. Royal Brompton \& Harefield NHS Foundation Trust. New opportunities for the treatment of hyperlipidaemia: initiation of PCSK9 monoclonal antibodies utilising a multi-disciplinary approach. NICE. Available from: https://www.nice.org.uk/sharedlearning/newopportunities-for-the-treatment-of-hyperlipidaemia-initiation-ofpcsk9-monoclonal-antibodies-utilising-a-multi-disciplinary-approach. Accessed April 29, 2021.

33. Leeds Teaching Hospitals NHS Trust. Innovative medicines optimisation clinic for PCSK9 inhibitors \& statin intolerance. Available from: https://www.nice.org.uk/sharedlearning/innovative-medicinesoptimisation-clinic-for-pcsk9-inhibitors-statin-intolerance. Accessed April 29, 2021.

34. Wood FA, Howard JP, Finegold JA, et al. N-of-1 trial of a statin, placebo, or no treatment to assess side effects. $N$ Engl $J$ Med. 2020;383(22):2182-2184. doi:10.1056/NEJMc2031173

35. Aubiniere-Robb L, Dickerson JE, Brady AJB. Lipid testing and treatment after acute myocardial infarction: no flags for the flagship. Br J Cardiol. 2019;26:141-144. doi:10.5837/bjc.2019.041

36. Danese MD, Pemberton-Ross P, Catterick D, Villa G. Estimation of the increased risk associated with recurrent events or polyvascular atherosclerotic cardiovascular disease in the United Kingdom. Eur $J$ Prev Cardiol. 2020;1-10. doi:10.1177/2047487319899212

37. Drexel H, Coats AJS, Spoletini I, et al. ESC position paper on statins adherence and implementation of new lipid-lowering medications: barriers to be overcome. Eur Heart $J$ Cardiovasc Pharmacother. 2019. doi:10.1093/ehjcvp/pvz079

38. Rosenson RS, Baker S, Banach M, et al. Optimizing cholesterol treatment in patients with muscle complaints. $\mathrm{J} \mathrm{Am}$ Coll Cardiol. 2017;70(10):1290-1301. doi:10.1016/j.jacc.2017.07.752

39. Connolly SB, Kotseva K, Jennings C, et al. Outcomes of an integrated community-based nurse-led cardiovascular disease prevention programme. Heart. 2017;103(11):840-847. doi:10.1136/heartjnl2016-310477

40. Waters DD, Boekholdt SM. An evidence-based guide to cholesterollowering guidelines. Can $J$ Cardiol. 2017;33(3):343-349. doi:10.1016/j.cjca.2016.10.019 
41. Graham I, Shear C, De Graeff P, et al. New strategies for the development of lipid-lowering therapies to reduce cardiovascular risk. Eur Heart J Cardiovasc Pharmacother. 2018;4(2):119-127. doi:10.1093/ehjcvp/pvx031

42. Soran H, Kwok S, Adam S, Ho JH, Durrington PN. Evidence for more intensive cholesterol lowering. Curr Opin Lipidol. 2017;28 (4):291-299. doi:10.1097/MOL.0000000000000433

43. National Institute for Health and Care Excellence (NICE). Cardiovascular disease prevention overview. Available from: https:// pathways.nice.org.uk/pathways/cardiovascular-disease-prevention. Accessed April 29, 2021.
44. Patient decision aid. Cardiovascular disease: risk assessment and reduction, including lipid modification. Guidance. NICE. Available from: https://www.nice.org.uk/guidance/cg181/resources/patient-deci sion-aid-188102. Accessed April 29, 2021.

\section{Publish your work in this journal}

Vascular Health and Risk Management is an international, peerreviewed journal of therapeutics and risk management, focusing on concise rapid reporting of clinical studies on the processes involved in the maintenance of vascular health; the monitoring, prevention and treatment of vascular disease and its sequelae; and the involvement of metabolic disorders, particularly diabetes. This journal is indexed on PubMed Central and MedLine. The manuscript management system is completely online and includes a very quick and fair peerreview system, which is all easy to use. Visit http://www.dovepress. com/testimonials.php to read real quotes from published authors. 